\title{
Temporal resolution of successive brief stimuli for the tongue and hand
}

\author{
LINDA PETROSINO and DONALD FUCCI \\ Ohio University, Athens, Ohio
}

\begin{abstract}
The temporal resolving power of the tactile sensory system was examined by obtaining fusion thresholds for a two-pulse vibratory stimulus. Fusion thresholds were obtained from the tongue and thenar eminence of the hand for 24 adult subjects. The means of the fusion thresholds for the tongue $(24.63 \mathrm{msec})$ for the thenar eminence $(24.73 \mathrm{msec})$ were found to be statistically similar. The similarity in fusion thresholds for the two test sites suggests that for this suprathreshold task the tactile modality responds as a whole, regardless of body location. Results are discussed with respect to cutaneous fusion values already reported in the literature.
\end{abstract}

More than a century of research has been performed on the temporal resolution of major sensory modalities. One of the earliest and most extensive investigations dealing with temporal acuity in the sensory modalities was reported by Exner in 1875 (cited in Hirsh \& Sherrick, 1961). Exner used clicks and brief flashes of light to examine temporal acuity for audition and for vision, respectively. Exner found that, for vision, two brief flashes of light resolved (fused or became one) at approximately $40 \mathrm{msec}$ and that only $2 \mathrm{msec}$ was necessary to resolve two auditory clicks. Recent literature has continued to suggest that a sensitive measure of sensory system acuity is its ability to temporally resolve stimuli. More specifically, a threshold of fusion (successiveness) may be obtained by determining how close together in time two stimuli may occur and yet be perceived as only one stimulus.

For the sense of touch, Uttal (1959) obtained estimates bewteen 10 and $45 \mathrm{msec}$ as temporal resolution values for electrocutaneous stimuli. Rosner (1961) also employed electrocutaneous paired shock to explore the fusion threshold for the skin. The values reported by Rosner for the fusion of successive electrocutaneous stimuli fell between 15 and $40 \mathrm{msec}$. Using mechanical taps, Gescheider $(1966,1967)$ measured temporal acuity by looking at the time necessary for resolving two clicks both auditorily and cutaneously. The cutaneous values that he reported were about $10-12.5 \mathrm{msec}$, depending on the locus of stimulation. Geldard and Sherrick (1971) investigated temporal acuity through the resolution of brief cutaneous stimuli with a "modified stimulator" and the implementation of a forcedchoice discrimination psychophysical procedure. The fusion values recorded approached 3-5 msec. Gescheider (1974) replicated the Geldard and Sherrick experiments with a similar experimental design and reported an average fusion threshold of $5.5 \mathrm{msec}$.

The authors' mailing address is: School of Hearing and Speech Sciences, Ohio University, Athens, Ohio 45701.
The research that has been done in temporal acuity measurement within the cutaneous system has offered little or no uniformity in equipment or methodology. The results have been measures of temporal acuity that differ depending on the way that acuity has been assessed, as well as on the criteria set for tactile fusion (Hirsh \& Sherrick, 1961). To date, temporal fusion values for the cutaneous system are not well established and are totally nonexistent for any of the oral structures. The purpose of this study was to acquire additional information concerning the temporal resolving power of the tactile sensory system by determining the threshold of fusion for the tongue (an oral structure) and the thenar eminence of the hand (a nonoral structure) through the use of a two-pulse vibrotactile stimulus.

\section{METHOD}

\section{Subjects}

Twenty-four undergraduate students (12 women and 12 men) were selected randomly from a group of 90 students enrolled in an introductory speech and hearing class. Ages ranged from 19 to 22 years (mean age $=19.75$ years) for the men and from 18 to 22 years (mean age $=19.33$ years) for the women. All subjects received credit points for participation. The subjects were screened by interview, in which they were asked about present and past major illnesses, past or present medications, hearing, vision, other senses, motoric function, and coordination. Only those subjects who presented an overall profile of good present general health were employed in the actual experiment. None of the subjects had had prior experience with psychophysical testing or were familiar with the vibrotactile instrumentation used in this study.

\section{Apparatus}

The vibrotactile instrumentation consisted of a stimulus unit and a measurement unit. The stimulus unit was composed of Coulbourn solid-state logic modules. These modules can generate pulsed vibratory signals that vary in frequency, intensity, and temporal characteristics. An examiner interface consisted of a hand-held push button. Depressing the button delivered the signal to the test structure as preset by a signal pulse generator and other modules. The signals delivered from the stimulus control unit drove a minielectromagnetic vibrator. The measure- 
ment unit included an accelerometer, a cathode follower, a microphone amplifier, and a storage oscilloscope. A narrowband noise generator was used to present auditory masking at $70 \mathrm{~dB}$ HTL to subjects to prevent them from hearing the pulsed stimulus being generated. A detailed description of the vibrotactile instrumentation and procedure can be found in a recent investigation by Petrosino (1983).

\section{Stimulus}

Vibrotactile fusion thresholds were obtained from the anterior midline dorsal surface of the tongue and the thenar eminence of the preferred hand for each subject through the use of a two-pulse stimulus. The pulses generated were 1-msec square-wave electrical pulses, with the second sequentially generated pulse having a variable time of onset after the first pulse. Since it had been suggested that intensity imbalances between the pulses alter the threshold of fusion (makes fusion thresholds poorer), the stimulus consisted of two equally intense pulses (Gescheider, 1967). The pulses were presented at $35 \mathrm{~dB}$ SL, a moderately intense signal that has been employed successfully in prior cutaneous research (Gescheider, 1966; Petrosino, 1983).

\section{Procedure}

Each subject was seated in an adjustable chair and was asked to extend the appropriate test structure against a rigidly mounted plastic disk. A hole in the disk allowed access for the probe to be lowered through the center of the disk to touch the test surface. To maintain constant pressure of the vibrator probe on the test structure for all subjects, the probe was lowered until a voltmeter recorded contact. It was then lowered $1 \mathrm{~mm}$ farther into the structure from the point of recorded contact. The contactor at the end of the probe had an area of $.128 \mathrm{~cm}^{2}$. The hole in the disk had a constant space of $1 \mathrm{~mm}$ around the contactor.

Training was given to familiarize each subject with instrumentation and testing procedures. Prior to actual fusion threshold testing, baseline thresholds of sensitivity for a two-pulse stimulus were obtained to provide a reference for the 35-dB SL intensity level. Six readings (in millivolts) were obtained, and the mean of the last three readings was accepted as the baseline threshold. The millivolt readings were then converted to decibels re 1 -micron peak. The subjects were seated in an adjustable chair and positioned to be able to easily and comfortably assume the test position (extension of the tongue or thenar eminence against the plastic disk). The order of test site presentation was randomized. The subjects were instructed to respond by raising their nontest hand as soon as they had detected the stimulus on the structure being tested. An ascending method of limits was used to obtain threshold-of-sensitivity values. Once the thresholds of sensitivity had been obtained, the 35-dB SL was present and utilized throughout the testing of fusion threshold for a particular structure.

Subsequent to adequate training and threshold data collection, the subjects were instructed for fusion threshold testing. After the subjects understood the task, the actual testing was initiated. The two-pulse stimulus was presented at $35 \mathrm{~dB}$ SL, starting with an interstimulus interval of $40 \mathrm{msec}$. This interval was selected as a starting point because it was well above the fusion point already established in the literature for cutaneous sites (Gescheider, 1966, 1967; Hirsh \& Sherrick, 1961; Petrosino, 1983). Each subject was instructed to assume the test position for the structure to be tested when the auditory masking was introduced. Three presentations of the paired stimulus signal were given before judgment was made as to whether the stimulus was perceived as being one or more than one (Gengel \& Hirsh, 1970). When the masking was turned off, the subject made a decision.

The testing was started at such a level that the subject was able to perceive the stimulus as two discrete presentations. This time interval was then decreased until a fusion point (the stimulus was perceived as one) had been reached. Thornbury and Mistretta (1981) referred to this type of event as a reversal point. When a fusion point had been reached, the time interval was increased until the subject reported the presence of two stimuli (reached another reversal point). The test procedure was ended when four reversal points had been obtained. The mean of the four reversals was accepted as the critical fusion threshold (in milliseconds). This exact experimental procedure was followed for the collection of all fusion thresholds for each subject and test site

\section{RESULTS AND DISCUSSION}

The data from this investigation were subjected to an analysis of variance for a two-factor design for repeated measures (Bruning \& Kintz, 1977). The overall analysis indicated no statistically significant differences between subjects when grouped by sex, test sites themselves, or between sex $x$ test site. Analysis was conducted at the .05 alpha level.

Since no statistical difference was seen between male and female subjects, the data were combined for all 24 subjects. The mean fusion threshold for the tongue was $24.63 \mathrm{msec}$, with a standard deviation of 9.92 . The mean fusion threshold for the thenar eminence was $24.73 \mathrm{msec}$, with a standard deviation of 9.12. Large standard deviations for both the tongue and thenar eminence indicate a large difference in the fusion threshold response across subjects. This type of variation in psychophysical functions is often due to the behavioral difference in how the subjects perceive and respond to the stimuli. It is possible that the subjects employed different response criteria for establishing their fusion thresholds, which would account for the variance. The statistical analysis suggests that fusion thresholds for a two-pulse stimulus are similar for the tongue and thenar eminence and that fusion threshold are the same for men and women between the ages of 18 and 22 years.

The vibrotactile fusion task is not an absolute threshold judgment, but rather a suprathreshold acuity task. It has been well documented throughout the tactile literature that the skin varies in its absolute threshold of sensitivity at various body sites, presumably depending on the density of innervation and type of mechanoreceptors found there (Kenshalo, 1978). Even though the thenar eminence and the tongue produced different sensitivity thresholds, the fusion thresholds were similar. This similarity in fusion thresholds for both test sites suggests that, for this suprathreshold task, the tactile modality responds as a whole, regardless of body location. These results are compatible with recent suprathreshold work of Fucci and Petrosino (1983). Fucci and Petrosino investigated the scaling responses for the tongue, the thenar eminence of the right hand, and the distal pad of the middle finger of the right hand. The results showed that the three different body sites produced similar magnitude production curves. Therefore, the three different body sites within the same cutaneous 
sensory system produced compatible functions just as the body locations produced similar fusion thresholds in this study. The similarity appears to be inherent in a suprathreshold (central) task but not in a threshold of sensitivity (peripheral) task.

The 24.63- and 24.73-msec mean fusion thresholds for the tongue and the hand obtained from this study fall within a range that has been reported from studies using electrocutaneous shock. These values are twice as large as those reported from studies employing mechanical vibration. One possible reason that the fusion thresholds from this study are twice as large as those from other fusion studies employing mechanical vibration is the relatively small contactor employed. A recent attempt has been made to investigate systematically the effect of different contactor sizes on the suprathreshold task of magnitude production on the lingual structure as well as the thenar eminence (Fucci, Harris, \& Petrosino, 1983). Three different contactor sizes were used to obtain curves for the thenar eminence and the tongue. The use of larger contactors produced lower magnitude functions than those produced with smaller contactors. Therefore, the number of sensory units activated by the size of the contactor is an important variable in establishing vibratory sensation magnitude functions. Gescheider $(1966,1967)$ used a contactor measuring $.32 \mathrm{~cm}^{2}$, which was 2.5 times larger than the contactor of $.128 \mathrm{~cm}^{2}$ used in the present study. The contactor in the vibratory system employed by Geldard and Sherrick (1971) was even larger than that of Gescheider (1966, 1967), a fact that, along with the forced-choice methodology, caused the fusion thresholds to be even lower. The fact that the fusion thresholds from the present study were twice as large as those reported by earlier investigators adds support to the observation that the peripheral and central neural mechanisms of the cutaneous system have sufficient specificity to make spatial (contactor-size) as well as temporal discriminations (Telage \& Warren, 1977; Verrillo, 1966; Verrillo \& Chamberlain, 1972).

Another possible explanation for the relatively large fusion thresholds obtained in this study is the instrumentation setup. The instrumentation setup employed in this study was different from that used for other mechanical vibration fusion studies. Other researchers have typically employed two vibrators to deliver pulses in succession to the test site. The present study employed only one vibrator, since the signal pulse generator and the gating devices in the system were capable of delivering the pulses in succession. The lingual structure as a test site also necessitated the use of the single vibrator specifically designed to test the relatively small lingual structure.
The fusion threshold technique and instrumentation employed in this study appear to provide a viable way of using a suprathreshold task to assess the temporal acuity of the normal tactile sensory system. A problem that exists in the tactile fusion literature is the discrepancies in fusion threshold values reported by different investigators. Further research is necessary to resolve these differences in order to eventually obtain a stable body of literature in the field of somatosensory research.

\section{REFERENCES}

Bruning, J. L., \& Kintz, B. L. (1977). Computational handbook of statistics. Glenview, IL: Scott Foresman.

Fucci, D., Harris, D., \& Petrosino, L. 1983). The effect of contractor area on vibrotactile magnitude function exponents for the tongue and hand. Bulletin of the Psychonomic Society, 21, 400-402.

Fucci, D., \& Petrosino, L. (1983). Lingual vibrotactile sensation magnitudes: Comparison of suprathreshold responses for the tongue and hand. Journal of the Acoustical Society of America, 74, 351-353.

Geldard, F. A., \& Sherrick, C. E. (1971). Cutaneous temporal acuity. Princeton Cutaneous Reports, 18, 8-10.

Gengel, R. W., \& Hirsh, I. J. (1970). Temporal order: The effect of single versus repeated presentations, practice, and verbal feedback. Perception \& Psychophysics, 7, 209-211.

Gescheider, G. A. (1966). Resolving of successive clicks by the ears and skin. Journal of Experimental Psychology, 71, 378-381.

Gescheide R, G. A. (1967). Auditory and cutaneous resolution of successive brief stimuli. Journal of Experimental Psychology, 75, 570-572.

Gescheider, G. A. (1974). Temporal relations in cutaneous stimulation. In F. A. Geldard (Ed.), Cutaneous communication systems and devices. Austin, TX: Psychonomic Society.

Hirsh, I. J., \& Sherrick, C. E. (1961). Perceived order in different sense modalities. Journal of Experimental Psychology, $62,423-432$.

Kenshalo, D. R. (1978). Biophysics and psychophysics of feeling. In D. R. Kenshalo (Ed.), Handbook of perception. New York: Academic Press.

Petrosino, L. (1983). Cutaneous temporal resolution assessed by the critical fusion threshold technique. Unpublished doctoral dissertation, Ohio University, Athens, Ohio.

Rosner, B. S. (1961). Neural factors limiting cutaneous spatiotemporal discriminations. In W. R. Rosenblith (Ed.), Sensory communication. Cambridge, MA: M.I.T. Press.

Telage, K., \& Warren, J. (1977). Spatial summation effects on lingual vibrotactile thresholds. Perceptual \& Motor Skills, 44, 1179-1185.

Thornbury, J. M., \& Mistretta, C. M. (1981). Tactile sensitivity as a function of age. Journal of Gerontology, 36, 34-39.

UTTAL, W. R. (1959). A comparison of neural and psychological responses in the somesthetic system. Journal of Comparative Physiology \& Psychology, 52, 485.

Verrillo, R. T. (1966). Effect of spatial parameters on the vibrotactile threshold. Journal of Experimental Psychology, 71, 570-575.

Verrillo, R. T., \& Chamberlain, S. C. (1972). The effect of neural density and contactor surround on vibrotactile sensation magnitude. Perception \& Psychophysics, 17, 117-120.

(Manuscript received for publication December 30, 1983.) 